\title{
EL TRABAJO SOCIAL Y LAS NUEVAS FORMAS DE REORGANIZACIÓN DEL CUIDADO. UNA APROXIMACIÓN A PROPÓSITO DE LA LEY DE DEPENDENCIA
}

\author{
Social work and the new ways of reorganising care. \\ A consideration of the Dependent Care Law
}

\author{
Virginia Fuentes GUTIÉRREZ* \\ Jesús Muyor RODRÍGUEZ** \\ ZAHIRA GALINDO ROMERO***
}

\section{Resumen}

En España se está produciendo en los últimos años una reestructuración en la gestión de las responsabilidades del cuidado, influenciada, principalmente, por la aprobación de la Ley 39/2006 de Promoción de la Autonomía Personal y Atención a las personas en situación de dependencia (LAAD, en adelante) que conlleva la introducción de ¿nuevos? actores en su ejecución.

* Virginia Fuentes Gutiérrez

Universidad de Jaén

Departamento de Trabajo Social y Servicios Sociales

C/ Profesor Tierno Galván, 10. Esc. B. Bajo B. 14014 Córdoba

Teléfono: 679392003

virginiafuentesgutierrez@hotmail.com,vfuentes@ujaen.es

* * Jesús Muyor Rodríguez

MediCall® Servicios Sociosanitarios, S.L.

Avd. Mariano Hernández. Apartado de Correos n. ${ }^{\circ} 481.04740$ Almería

Teléfono: 9503264 87/692032659 Fax: 950326487

jesusmuyor@medicall.es

*** Zahira Galindo Romero

Universidad de Jaén

C/ Padre Manjón, 8. 14400 Pozoblanco (Córdoba)

Teléfono: 654201387

zahiragalindo@hotmail.com 
Con el presente texto, desde la disciplina y práctica del Trabajo Social, recogemos algunas reflexiones sobre los modos en los que está siendo acometida dicha reorganización en el actual sistema de cuidados, atendiendo especialmente al contexto andaluz. Dirigimos nuestra atención hacia la aplicación e impactos que las vigentes políticas referentes al cuidado están teniendo sobre la práctica profesional y modalidades de provisión de cuidados.

Por último, pretendemos abordar algunas de las situaciones derivadas de la implantación de la LAAD para plantear posibles controversias y dilemas que se suscitan y que inevitablemente condicionan el quehacer profesional del Trabajo Social.

Palabras clave: cuidados, Ley 39/2006 de Promoción de la Autonomía Personal y Atención a las personas en situación de dependencia (LAAD), Trabajo Social, dependencia.

\section{Abstract}

In recent years, Spain has begun to restructure how the responsibilities of care are managed, mainly as a result of the 2006 Personal Autonomy and Dependent Care Law (PADCL), with new(?) actors becoming involved in its implementation. Based on both the theory and the practice of social work, this paper reflects on how this reorganisation is being carried out in the current care system, particularly in Andalusia, examining how current care policies are applied and what effects they are having on professional practice and the ways in which care is provided. The dissertation seeks to analyse some of the situations resulting from the implementation of the PADCL, and to consider possible controversies and dilemmas that may arise that will inevitably have an affect on the social work profession.

Keywords: care, Personal Autonomy and Dependent Care Law (PADCL), Social Work, Dependent Care.

\section{Introducción}

El modo en que se viene dando respuesta a las necesidades de cuidados de las llamadas personas dependientes parece experimentar cambios (isignificativos?) en España, en sus maneras de concebirlo, legislarlo y gestionarlo, tanto a nivel institucional como familiar.

Podemos considerar que la falta de autonomía funcional que supone la situación de dependencia no constituye una situación de necesidad nueva, sin embargo lo que le añade un perfil novedoso a este fenómeno es su generalización, en cuanto al número de personas afectadas, la intensificación en efectos y tiempo y, sobre todo, la conciencia respecto de la ineficacia de las formas tradicionales de afrontarla (supeditada a la solidaridad, al apoyo familiar, y centrada sólo en los factores individuales de la persona), dejando de lado el cariz social. Esta situación de dependencia, vista también desde un plano social, implica, por otro lado, que la atención normativa no se proyecte únicamente y exclusivamente en el ámbito de la asistencia sanitaria. El objetivo 
básico de cualquier protección contra las situaciones de dependencia debe ser, en primer lugar, el hacer desaparecer, en el mayor grado posible, aquellas circunstancias sociales que pueden provocarlas o aumentarlas y, a partir de ahí, proporcionar a la persona la asistencia, ayudas y auxilios que necesita para poder realizar, adecuada y dignamente, las actividades de la vida diaria para las que requiere ayuda (De Asís y Palacios, 2007: 19-20).

En esta directriz, el sistema público español de Servicios Sociales ha sufrido, en los últimos años, una reestructuración que ha provocado la unificación del sistema de protección a las situaciones de dependencia en el marco de la LAAD. Los/as trabajadores/as sociales han sido, y continúan siendo, un actor importante en la implementación de los servicios y recursos derivados del Sistema para la Autonomía y Atención a la Dependencia (SAAD, en adelante).

Desde esta premisa es desde la que se analiza dicha implementación de la Ley que ha ido configurando una serie de desconciertos, dilemas y retos en lo que se denomina un derecho subjetivo, individual y universal a recibir atención necesaria cuando se está en situación de dependencia.

El texto de este artículo se divide en tres apartados. En primer lugar, nos preguntamos sobre el amplio campo relativo al desarrollo de la dimensión socio-política e ideológica presentado en dicha Ley y sus impactos sobre la (re)producción de las desigualdades de género, vinculadas con el mantenimiento del rol subordinado de las mujeres, la división sexual del trabajo, la generización que atraviesa el mercado de trabajo derivado del cuidado o la invisibilidad y condiciones de precariedad en la que se encuentran las mujeres inmigrantes cuidadoras. Nos interesa especialmente enmarcarlo en lo que se define como mercantilización y externalización del trabajo doméstico y de cuidados, así como detenernos en el papel que juegan los agentes sociales que parecen implicarse (Estado, mercado, Tercer Sector y familia) en la llamada reorganización del cuidado. Trataremos de hacer emerger algunas de las posibles contradicciones que se activan. En un segundo lugar, dirigiremos nuestra atención hacia la dimensión deontológica de nuestra actividad profesional, con el objetivo de cuestionarnos e interrogarnos sobre el papel desempeñado por los trabajadores sociales como meros técnicos de gestión o, por el contrario, como posibles actores implicados en el diseño de la política pública y en la evaluación de las situaciones de dependencia. Por último, nos centraremos en la trascendencia y connotaciones prácticas que la implantación de la LAAD está teniendo para el Trabajo Social y los Servicios Sociales. Aspectos como la burocratización del servicio, reajustes de las prestaciones, dilemas éticos, la (escasa) formación de los profesionales o la posible desatención de otras áreas de acción de los servicios sociales son limitaciones y retos que nos plantean el 
debate, abierto y pendiente, sobre el papel desempeñado en la actualidad por los profesionales del Trabajo Social.

Con estas tres dimensiones tenemos el propósito de recapacitar sobre la oportunidad que esta nueva reestructuración de cuidados puede dar a lugar, mostrando una imagen del Trabajo Social en concordancia con los principios propios de esta profesión.

\section{Dimensión socio-política e ideológica}

2.1. Una gestión pluralista. El papel de los agentes sociales en el proceso de reorganización de los cuidados

La LAAD surge en un momento de profundo cambio social (lo que se ha denominado también "Crisis de los cuidados $»^{1}$ ) caracterizado por políticas neoliberales que impulsan la globalización y que generan cada día mayor precariedad en el empleo, en la vivienda, en la alimentación, en las condiciones de salud, en las prestaciones sociales y en las formas de vida. La quiebra con el modelo previo de provisión de cuidados -apoyado en una estricta división sexual del trabajo y en la imposición de la familia nuclear heterosexual como norma social-, que era la base del sistema económico (Pérez de Orozco y Baeza Gómez, 2006: 24), ha propiciado que se empiece a pensar en otras formas de reorganización y modelos de cobertura del cuidado.

Podría decirse que caminamos hacia una nueva gestión del cuidado en la que siguen participando agentes tradicionales, principalmente familias, y a los que se van a incorporar otras fuentes de gestión del cuidado sustentadas principalmente a través del Estado y del mercado. La labor del cuidado se convertiría así en una responsabilidad compartida, al menos parcialmente, en la que participan a nivel macro varias esferas interconectadas entre sí y que incluso a veces intervendrían simultáneamente, precisamente por la insuficiencia de los recursos aportados por cada una de ellas.

Dicho de otro modo, dado el bajo nivel de cobertura existente de servicios sociales, a pesar del innegable crecimiento de los últimos años, sobre todo de atención y rehabilitación (Centros de día y de noche) y domiciliarios (Ayuda a domicilio), además de otros de tipo intermedio (estancias temporales y de respiro) y alternativos (viviendas tuteladas, domicilios de acogida) (Rodríguez Cabrero, 2007), son las familias, en ocasiones, las que se ven obligadas a superponer los recursos públicos y privados de manera que puedan obtener la

1. Para saber más, ver Río, 2003; Colectivo Precarias a la deriva, 2004; Pérez Orozco, 2006a, Pérez de Orozco y Baeza Gómez, 2006; Vega, 2009. 
ayuda más completa posible de cara al cuidado de la persona en situación de dependencia. Así, si a una persona en situación de dependencia se le asignan formalmente un tipo de recurso en su PIA ${ }^{2}$, la familia por otro lado, si su nivel de renta se lo permite, podrá contratar en el mercado otro servicio de proximidad complementario para así garantizar el eficaz cuidado a su familiar.

Ante esta realidad, nos preguntamos ies previsible que se consolide un modelo dual de sociedad que acentúe las desigualdades sociales, en el que el nivel de renta determine la calidad de vida de las personas en situación de dependencia? Según Amaia Pérez y Paula Baena, por un lado se situarían los segmentos de población con suficiente poder adquisitivo para satisfacer la mayor parte de sus necesidades en base a la compra de servicios en el mercado. Y, por otra parte, se localizarían aquellos segmentos de población que no tienen ese nivel de consumo y sustituyen compra en el mercado por provisión gratuita y en muchas ocasiones residual de servicios (Pérez de Orozco y Baeza Gómez, 2006: 32).

Es preciso insistir en que dichas situaciones antagónicas, basadas en el tener o no suficiente poder adquisitivo como para satisfacer la necesidad del cuidado a través de un modo u otro (Estado o mercado), no deben ser vistas como excluyentes. En la realidad, como ya adelantábamos, surgen situaciones de compensación intermedias caracterizadas por la coexistencia de múltiples formas de cubrir la necesidad de cuidados. Como mecanismos intermedios que entrañan algún riesgo en su implantación destacamos entre otros:

- Las prestaciones monetarias para el cuidado informal, que si no se utilizan con el criterio de excepcionalidad ${ }^{3}$ por el que fueron creadas, podrían hacer demorar la construcción de una red pública de servicios sociales directa y concertada suficientemente, además de una perpetuación de la ayuda prestada en el ámbito familiar.

- La provisión gratuita de servicios suministrada por las ONGs evitaría situaciones de emergencia social, pero a su vez, podría evadir la responsabilidad pública del Estado.

2. Programa Individual de Atención (PIA), realizado por los Trabajadores sociales desde los Centros de Servicios Sociales Comunitarios, determinará las modalidades de intervención más adecuadas a sus necesidades de entre los servicios y prestaciones económicas previstos en la resolución para su grado y nivel.

3. En el caso andaluz de las 169368 prestaciones concedidas en la Comunidad andaluza a 1 de diciembre de 2009, 85021 (50,20\%) fueron destinadas a prestaciones económicas a cuidadores familiares y 3201 (1,89\%) prestaciones económicas fueron vinculadas a servicios concedidos, según datos del IMSERSO, [en línea], <http://www.imserso.es/ Presentacion/groups/imserso/documents/binario/estsisaad20091201.pdf>. Extraído el 14 de diciembre de 2009. 
- La ayuda invisibilizada de empleadas del hogar, en la mayoría de los casos mujeres inmigrantes, que debido a su situación de vulnerabilidad social y jurídica se inserta en el mercado del cuidado de una forma irregularizada (Colectivo IÓE, 2001). Situación de la que se benefician los empleadores cuando el resto de recursos sociales no satisfacen sus necesidades o, si lo hacen, son demasiado costosas para su nivel de renta.

Estas opciones intermedias de reorganización del cuidado reflejan los mecanismos que tanto el Estado, mercado, organizaciones sociales y familia disparan para afrontar una situación de cuestionamiento del actual modelo de provisión de cuidados. Esto nos hace abrir algunas dudas sobre cuál es la verdadera ideología que sustenta a la LAAD y el peso que, pareciera, siguen asumiendo especialmente las familias en la legislación actual. En palabras de Rodríguez Cabrero «se podría pensar que la Ley de Dependencia parece más un reconocimiento del actual sistema social familista que una revolución social basada en la universalización de la acción protectora a través del Estado» (Rodríguez Cabrero, 2007: 74).

También nos parece importante empezar a reflexionar sobre el papel que están desempeñando los Servicios Sociales y las entidades del Tercer Sector y empresas de servicios en la construcción de este sistema de atención al cuidado que parece activarse.

\subsubsection{Servicios Sociales ${ }^{4}$}

Por un lado, vemos cómo se reconoce a los servicios sociales su papel como puerta de entrada al SAAD, delegándose, por tanto, una parte importante de la demanda de usuarios que genera la Ley de Dependencia. En contrapunto, cabe preguntarse sobre los recursos personales y materiales puestos en marcha para afrontar esta nueva competencia. En el informe «Perfeccionamiento de los Servicios Sociales en España», con motivo de la creación de la Ley de Autonomía y Dependencia se pone de manifiesto que «la red de equipos con que cuentan los servicios sociales que habrían de realizar esta labor en el área de discapacidad son insuficientes y se dedican sólo, salvo excepciones afortunadas, al reconocimiento administrativo de la discapacidad» (Casado y Fantova, 2007: 9).

Ante esta realidad, ¿cuál es el papel que están jugando los Servicios Sociales públicos en la gestión de la LAAD? ¿Realmente los recursos humanos y materiales se han aumentado como para hacer frente a esta demanda social?

4. Retoma el estudio de los Servicios Sociales en apartados posteriores, sobre todo en lo referido a la figura del trabajador social en dicha institución. 
¿La ampliación de recursos en esta área específica de lo social puede traer consigo un abandono del resto de áreas de atención? Planteamos aquí una serie de interrogantes abiertos a la reflexión, de aspectos sobre los que indagar e investigar para suscitar el debate sobre cómo se está gestionando el SAAD en el contexto público.

De otro modo, es preciso señalar que la creación de un sistema de Atención a la Dependencia, tal y como se ha configurado en nuestro país, implica no solo la participación del Estado, sino también que éste se apoye en una red concertada y privada de organizaciones del Tercer Sector por un lado y empresas dedicadas a la prestación de servicios sociales por el otro.

\subsubsection{Organizaciones del Tercer Sector}

En nuestro intento por aglutinar una serie de ideas, como resultados de plantearnos algunos interrogantes derivados de la nueva gestión del cuidado a partir de la aprobación de la LAAD, nos preguntamos en esta ocasión sobre el papel que están desempeñando las entidades del Tercer Sector en el proceso de creación del SAAD.

Concretamente se define al Tercer Sector en la LAAD como (artículo 2): «Organizaciones de carácter privado surgidas de la iniciativa ciudadana o social, bajo diferentes modalidades que responden a criterios de solidaridad, con fines de interés general y ausencia de ánimo de lucro, que impulsan el reconocimiento y el ejercicio de los derechos sociales».

Las entidades no lucrativas se caracterizan por las numerosas y diversas formas que adquieren ${ }^{5}$, surgiendo así ONGs, asociaciones, entidades voluntarias, instituciones privadas sin fines de lucro (IPSFL) y organizaciones humanitarias, entre las más comunes. Pero lo que parece de algún modo caracterizar a dichas entidades es su objeto social y carácter altruista. Dicha delimitación nos orienta y limita el campo dentro del universo asociativo, pero resulta difícil la calificación de tipologías de asociaciones, dado que existe un segmento de organizaciones e iniciativas de objeto social que se aproxima a la esfera lucrativa del mercado (Zurdo Alaguero, 2007).

Es en este debate en el que nos cuestionamos el posible acercamiento de las entidades no lucrativas a identidades cada vez más circunscritas a su papel de proveedoras de servicios sociales (principalmente servicios de ayuda a la dependencia y servicio doméstico). Como advierte Luis Enrique Alonso «las ONG muchas veces representan más intentos de profesionalización de colectivos que no pueden entrar en el mercado de trabajo, búsqueda de beneficios

5. Para saber más, véanse Zurdo Alaguero (2007) y Alonso Benito (2000). 
y de subvenciones utilizado el señuelo del bienestar social...» (Alonso Benito, 1998: 167).

En el mismo sentido, Martínez Román, Mira-Perceval Pastor y Pedero Bellido (1996: 235) observan la aparición y proliferación en los últimos años de empresas enmascaradas de ONGs [...] «constituidas, en realidad, por profesionales que, al elegir esta forma organizativa, pueden acceder a subvenciones de la Administración pública y a las correspondientes desgravaciones fiscales».

En conclusión, nos parece interesante repensar en el rol que ¿empiezan? a jugar las organizaciones del Tercer Sector, que pareciera más acercarse a la lógica del mercado en su papel como proveedoras de servicios, gestionando recursos a la sociedad, en detrimento de su papel como espacio independiente generador de reivindicación social. Nos encontramos pues ante la eclosión de un asociacionismo activo y voluntario, difícilmente descifrable usando la división tradicional entre Estado y Sociedad Civil (Alonso Benito, 2000).

\subsubsection{Empresas prestadoras de Servicios Sociales}

Las empresas sociales empezaron a proliferar en nuestro país como conocedoras de la futura demanda ${ }^{6}$ que sobre ellas recaería a partir de la aprobación de la LAAD. Recordemos que una empresa proveedora de servicios sociales responde a la lógica del beneficio empresarial, beneficio que obtienen a partir de la oferta de prestaciones y servicios sociales en pro del bienestar social, pero plenamente insertas en el mercado y por ende en la búsqueda del beneficio económico. Esta búsqueda de beneficio económico hace que las reglas del juego empresarial se asienten en la minimización de costes y ampliación de beneficios. Lógica que parecería no casar con las buenas intenciones de la LAAD al hablar de un derecho universal y de calidad para todos los ciudadanos ${ }^{7}$. Ante

6. Esa proliferación de empresas dedicadas a la prestación de servicios sociales viene fuertemente condicionada por la LAAD que promueve que "Cuando las personas en situación de dependencia no puedan acceder a un recurso público o concertado, tendrán una ayuda económica para tener acceso a un dispositivo privado, para lo que se consignarán 15,1 millones de euros», [en línea], <http://www.juntadeandalucia.es/ fundaciondeserviciossociales/es/noticias/not_071106/wfnews_view_pub>.

7. En el Artículo 3 de la LAAD se establecen los principios inspiradores que sustentan la ley, entre los cuales encontramos: «2. La universalidad en el acceso de todas las personas en situación de dependencia, en condiciones de igualdad efectiva y no discriminación, en los términos establecidos en esta Ley. 10. La calidad, sostenibilidad y accesibilidad de los servicios de atención a las personas en situación de dependencia y 13. La participación de la iniciativa privada en los servicios y prestaciones de promoción de la autonomía personal y atención a la situación de dependencia». 
esta situación cabe preguntarse si realmente la empresa privada va a asumir los costes derivados de establecer un servicio universal y de calidad aunque implique disminuir su cuantía de beneficio económico. Recordemos en este punto que las licitaciones públicas se realizan a un precio muy bajo. Esta imposición administrativa (precio del concurso público) adquiere un valor muy importante para que las empresas concursen en la gestión del servicio. De esta manera, son las empresas quienes tienen que adaptar el precio del servicio a las exigencias impuestas por la administración (precio muy bajo). Así, las empresas, en muchos casos, se ven forzadas a limitar la calidad del servicio para poder adecuarse al precio que fija la administración en el concurso público de gestión de los servicios.

Por otra parte, y enlazando con las entidades del Tercer Sector, nos planteamos lo siguiente: ¿se estrecha cada vez más la diferencia entre empresas de Servicios Sociales y Entidades del Tercer Sector? ¿Dónde queda el carácter garantista de la LAAD si la gran parte de su gestión (pública y/o privada) se encuentra mercantilizada? ¿Existen protocolos de seguimiento real, eficiente y eficaz sobre la gestión concertada?

\subsection{El papel de la familia y la mujer: ¿crisis del modelo tradicional?}

Como hemos introducido ya en el apartado anterior, en la LAAD se recogen una pluralidad de formas para cubrir la necesidad de cuidados: a través de entidades públicas, ONGs y entidades asociativas, en el sector privado con ánimo de lucro y en el ámbito familiar. Es precisamente en este último en el que ahora nos detendremos insistiendo en nuestra idea de plantear posibles contradicciones, aspectos interrogantes y dudas que parecen brotar.

Las investigaciones del IMSERSO sobre apoyo informal -realizadas en 2009- ponen de relieve que la gran mayoría de los cuidados que precisan las personas en situación de dependencia es asumida por las familias. Recordemos el dato en el caso andaluz de las 169368 prestaciones concedidas en la Comunidad andaluza a 1 de diciembre de 2009, de las que $85021(50,20 \%)$ fueron destinadas a prestaciones económicas para cuidadores familiares.

De igual modo, la feminización de la figura del denominado «cuidador principal familiar» es abrumadora en nuestro país según muestran los datos

Llegado el momento nos preguntamos: ¿Se podrían entrever en los principios inspiradores lógicas contradictoras? ¿Cómo casa el derecho universal y de calidad con el surgimiento de la iniciativa privada y empresarial cuyo objetivo, recordemos, es la búsqueda del beneficio económico? ¿Realmente es la empresa privada la que debe asumir los costes de un servicio de calidad y universal? ¿no debería ser el Estado el encargado de ofrecer estas garantías? 
del IMSERSO ${ }^{8}$, cabe destacar que si en 1994 el $83 \%$ del total de cuidadores eran mujeres, 15 años después ese porcentaje, por más que pueda resultar sorprendente, no solo no se ha modificado con el paso del tiempo, sino que llega incluso a incrementarse, ya que en 2009 el total de cuidadoras mujeres asciende a un $94 \%$ frente a un $6 \%$ de cuidadores hombres (IMSERSO).

Ante este análisis de los datos, es preciso que nos detengamos a reflexionar sobre la reproducción de la desigualdad de género que las políticas sociales y en concreto la LAAD parecen representar. Como afirman Pérez de Orozco y Baeza Gómez (2006: 14): «La Ley de dependencia se configura como una respuesta a una situación de emergencia social, derivada de la crisis de los cuidados, pero no como una herramienta para revertir injusticias históricas en el reparto de los trabajos».

La responsabilidad de cuidar, en el caso de las mujeres, se confunde con su rol principal en el mundo. Proporcionar cuidados a los demás ha sido un elemento central en la identidad femenina hasta hace bien poco. Las mujeres cuidan, se ha dicho muchas veces, incluso a costa de sí mismas, de su propia salud y bienestar (Vega Solís, 2009: 10).

La nueva legislación sobre dependencia propuesta por el Estado español parece apostar, como explicábamos, por un sistema de bienestar mixto en el que participan familia, Estado, mercado y voluntariado en distintas dosis. Pero surgen ciertas medidas como, por ejemplo, las prestaciones económicas dirigidas a retribuir a las personas cuidadoras de ámbito familiar, que nos hacen reflexionar sobre la supuesta liberación de las familias, y en especial de las mujeres, que dicha legislación promulgaba.

En este sentido, las prestaciones dirigidas a retribuir a las personas cuidadoras deberían considerarse prestaciones verdaderamente excepcionales, ya que el uso extendido de las mismas podría desincentivar el acceso y conservación del empleo de las mujeres al tiempo que contribuiría al estereotipo tradicional de asociar a los valores femeninos cuando se relacionan con la ética del cuidado (Rodríguez Rodríguez, 2005: 7).

Como podemos observar, es mucha la polémica en torno a la prestación económica por cuidados familiares, y el hecho de otorgar una cuantía económica visibiliza parcialmente la labor realizada de las familias pero ¿pudiera convertirse en un instrumento que sujete a muchas mujeres al ámbito familiar, inhibiéndolas de entrar en el mercado laboral?

8. Véase<http://www.imserso.es/Presentacion/groups/imserso/documents/binario/estsisaad 20091201.pdf>, extraído el 14 de diciembre de 2009. 
Otro aspecto sobre el que nos parece interesante reflexionar es la incorporación de las mujeres (in)migrantes al sector del cuidado (Agrela Romero, 2009a). Esta incorporación responde precisamente a esa necesidad presentada por las familias españolas como estrategia de organización en sus vidas cuando no encuentran apoyo por parte de las instituciones públicas y la esfera familiar presenta algunas circunstancias ${ }^{9}$ a partir de las cuales no puede hacerse cargo del cuidado de su familiar. Esta realidad individual/familiar y cotidiana tiene una repercusión a nivel macro, pues la demanda producida desde países como España para trabajar como empleadas de hogar implica a su vez que se produzca una oferta específica de mano de obra de mujeres en países emisores de (in)migración (Hochschild, 2001).

Unido a todo lo anterior, muchos son los factores que desestabilizan los niveles de oferta y demanda a los que tradicionalmente asistíamos. A modo de ideas: por un lado hemos presenciado la incorporación de la mujer española al mundo laboral. Además cada vez son más las mujeres con formación que eligen un empleo de mayor cualificación. De otro modo, la ocupación de empleada de hogar se presenta como una actividad laboral altamente precarizada y de escaso reconocimiento social. Las negociaciones sobre el reparto de tareas en el ámbito doméstico entre los hombres y las mujeres no se han producido de forma igualitaria, y surgen cada vez más nuevos modelos de familias, más complejos (familias monoparentales, familias reconstruidas, etc.) que requieren de nuevas estrategias de organización en el cuidado (Agrela Romero, 2009b).

La demanda de empleadas domésticas es cada vez mayor y dada su situación de precariedad del régimen laboral ${ }^{10}$ en nuestro país, es fácilmente predecible que sean las mujeres (in)migrantes las encargadas de ocupar este nicho laboral. Según el colectivo IOÉ, la ocupación del cuidado se convierte, hoy por hoy, en la principal puerta de entrada de las mujeres inmigrantes al mercado laboral español (IOÉ, 2005).

Recordemos que no sólo la demanda está ahí, sino que surge al mismo tiempo una internacionalización del mercado de trabajo acompañada de una

9. Entre ellas, destacan que la mujer esté inserta en el mercado laboral, que esté a cargo de otros miembros de la familia que también necesiten cuidados (por ejemplo, sus hijos), que no quiera encargarse de esa labor, o que la unidad familiar esté exclusivamente compuesta por varones.

10. El Real Decreto 1424/1985 de 1 de agosto por el que se regula la relación laboral de carácter especial del servicio de hogar permite, entre otras muchas cuestiones, que el pago de salario se pueda realizar en especia hasta un $45 \%$, que el tiempo de trabajo no supere las 40 horas semanales pero sin contabilizar los «tiempos de presencia» y que el contrato laboral se pueda realizar de forma oral, entre otras situaciones abusivas. 
aceleración de los flujos migratorios que hace que la inmigración femenina -fuertemente influenciada por la demanda de sus servicios- elija esta región como lugar de destino (Martínez Buján, 2009). Es en este punto donde nos preguntamos: $¿$ Es posible que aumenten las desigualdades entre mujeres según sus clases? ¿Qué papel juegan las mujeres (in)migrantes en la familia en la que ofrecen su servicio? ¿Cuáles son las condiciones en las que trabajan estas mujeres? ¿Qué repercusiones tiene la inmigración de mujeres en sus contextos de origen? ¿Qué valor se atribuye a la actividad de cuidar (laboral/ emocional)?

\section{Retos del Trabajo Social respecto a la dimensión deontológica}

En el actual paradigma ideológico de los Servicios Sociales públicos en España poco a poco entendemos que se trata de derechos universales y no de actividades o recursos especiales para colectivos vulnerables. Son áreas donde es necesaria, en general y para todo el mundo, una intervención profesional y, también, una protección pública (Fantova, 2009).

La LAAD se ha configurado bajo este modelo teórico de universalización y derechos sociales. No obstante, en el ejercicio diario del Trabajo Social, encontramos una serie de aspectos que permiten cuestionarnos el imaginario en el cual se estructura y desarrolla una ley de contenidos básicos que posiciona el quehacer del profesional del Trabajo Social, en la (re)estructuración del cuidado, bajo las órdenes técnico políticas y burocráticas.

Este debate entre una situación (cuestión política) y qué se debe hacer (cuestión ética) es una cuestión de sobra conocida en el Trabajo Social ${ }^{11}$ pero que vuelve a tomar relevancia en el tema en el cual lo encuadramos.

Como trabajadores/as sociales, transcurridos varios años desde la incorporación de esta ley, es el momento de reflexionar sobre el papel que estamos desempeñando y nuestra parte de responsabilidad en la (re)producción de las limitaciones y desconciertos que esta reestructuración del sistema de protección y reorganización del cuidado está desencadenando.

En el trámite de las situaciones de dependencia, en los Servicios Sociales comunitarios, hay tres situaciones en las que intervienen los/as trabajadores/ as sociales (Villalobos Santos, 2008): en el Servicio de Información, Valoración y Orientaciones, sobre la pertinencia, oportunidad, beneficios y trámites necesarios; en la elaboración del Informe Social y la Propuesta del Plan Individual de Atención (Propuesta PIA); y en el seguimiento de cada persona dependiente, una vez que el servicio o la prestación hayan quedado asignados

11. Para saber más, véase Moreno Pestaña, (2004). 
y, por la naturaleza de los mismos, impliquen que el dependiente continúa viviendo en su medio habitual. Partiendo de estas premisas, nuestras funciones no se están vinculando al activismo y al compromiso social que nos correspondería desempeñar. En las actuaciones de los/las trabajadores/as sociales que se derivan de la LAAD parece suscitar más «el hacer» que «el pensar», menospreciando la habilidad del Trabajo Social para desarrollar su propia identidad teórica y metodológica. Como expone Howe (1999), el pragmático se basa en el «sentido común» y suele significar una visión particular de ver las cosas. La actividad pragmática se rige por «si funciona, sirve» $\mathrm{y}$ «haz lo que pueda hacerse», sin cuestionarse que «lo que se puede hacer» no es lo mismo que «lo que se debe hacer» (Howe, 1999: 238-239).

El debate en estos términos se traduce en un Trabajo Social tecnócrata, en el que el profesional se especializa en los mecanismos institucionales frente a los modelos teóricos del Trabajo Social y la atención a los diferentes niveles de intervención (individual, familiar, grupal y comunitario):

«El reunir la documentación necesaria que viene establecida por el marco administrativo y gestionarla para solicitar un recurso en sí mismo, no es trabajo social. No obstante, pareciera que este es un tiempo en el que sin recurso no hay servicio. Pero si la función del trabajo social se redujera a esto, a gestionar la documentación para acceder a los recursos, cabe reconocer que para ello no se precisa ninguna diplomatura, sólo educación y conocer los papeles.» (Colom Masfret, 2005: 27)

En la actualidad, y en el desarrollo de la LAAD, estamos acometiendo el mismo papel pasivo que se le ha venido cuestionando históricamente al Trabajo Social. Centrado más en traducir la política social en servicio operativo que en aportar indicadores que contribuyan a la configuración de dicha política social, podríamos decir que el Trabajo Social se ha desarrollado más por la influencia del bienestar social y la política social en él que por la influencia contraria (De la Red, 1997: 100).

Precisamente, en este momento de reestructuración, en relación al sistema de protección de las situaciones de dependencia y la reorganización del cuidado, el Trabajo Social adquiere una actitud pasiva que se traduce en funciones relacionadas con la gestión de trámites y cumplimentación de formularios. Además, los Servicios Sociales continúan siendo organizaciones fuertemente jerarquizadas, burocratizadas, donde la figura profesional del trabajador social, principalmente, adquiere una función de respuesta frente a las demandas de la institución relegando, a un segundo plano, las necesidades de la ciudadanía.

Debemos recordar que el desarrollo e implementación de la LAAD se hace, principalmente, desde la base de los Servicios Sociales comunitarios. Esta 
vinculación de la política pública con la responsabilidad institucional de los Servicios Sociales recae sobre los trabajadores sociales que son los que sostienen la base del sistema y constituyen la referencia ciudadana e imagen pública de la misma. Esto sitúa a los trabajadores sociales ante el reto de perfeccionar su metodología de intervención, así como innovar, investigar y producir una creciente calidad profesional (Las Heras Pinilla, 2009: 171-172).

Por tanto, es nuestra responsabilidad visibilizar aquellas circunstancias que limitan el desarrollo efectivo, eficiente y real de un derecho subjetivo. Reconocer este papel y asumir una dimensión informadora y de crítica social trata de ser una propuesta que conecta la proyección de lo personal y lo disciplinar como poderoso argumento para modificar la situación que envuelve la profesión y enmarca la posición de los trabajadores sociales. Se trata pues de proyectar la realidad de las situaciones sociales estudiadas, de desarrollar una actividad de información y también de sensibilización que tendría como destinatarios a la sociedad en general, a los políticos y a los profesionales (Barbero, Feu y Vilbrod, 2007: 139-143).

\section{Retos del Trabajo Social respecto a la dimensión práctica}

Desde el Trabajo Social son múltiples y diversas las limitaciones que encontramos en la LAAD y que hacen necesario que el colectivo de profesionales que formamos esta disciplina profesional y científica manifieste.

En este aspecto encontramos que la práctica del Trabajo Social, en el desarrollo de la ley, se está vinculando, tal y como se ha mencionado con anterioridad, a los aspectos administrativos en la gestión de solicitud de reconocimiento del grado y nivel de dependencia, así como en la coelaboración del PIA. En ambos casos, los procedimientos y actuaciones vienen diseñadas desde un nivel técnico-político en el que el trabajador social no tiene margen de acción más allá de limitarse a rellenar los formularios y cuestionarios (pre) establecidos. Por el contrario, estos aspectos tan necesarios en la identificación de las necesidades en los cuidados se convierten en una tarea ardua de identificar, debido a las limitaciones de tiempo en los encuentros de valoración y evaluación, y difícil de reflejar en los registros cerrados que manejamos los profesionales.

Estas limitaciones administrativas se reflejan notablemente en el Informe Social y en el PIA, ya que las circunstancias que rodean a la persona en situación de dependencia (vivienda, relaciones sociales, comunidad, estructura y dinámica familiar, debilidades y puntos de apoyo...) pueden quedar incompletas en el diagnóstico técnico. 
Además de estas restricciones burocráticas que perjudican la práctica profesional de nuestra disciplina, se está produciendo, en multitud de ocasiones, un incumplimiento de los plazos previstos en los diferentes procesos administrativos. Esto puede estar proyectando una imagen de los Servicios Sociales como institución con excesivos procesos burocráticos que limita la resolución de las necesidades de la ciudadanía.

Por otra parte, cabe afirmar que estamos ante una ley de corte asistencial, enmarcada en el modelo médico-rehabilitador y con escasas referencias a la promoción de la autonomía personal. Además, podemos añadir que dicha ley «es ilegal y fuertemente discriminatoria para la mayoría del colectivo de personas con diversidad funcional (discapacidad)» (Foro de Vida Independiente, 2006), ya que sus principios no están en la línea de los establecidos por la Convención Internacional de la ONU, sobre los derechos de las personas con discapacidad, que posiciona a las personas con diversidad funcional como sujetos, ciudadanos titulares de derechos, participativos y responsables, que asumen la dirección total y completa de su vida personal y social.

Estos aspectos ideológicos condicionan la práctica profesional de nuestra profesión en la medida en que la red de servicios y equipos con que cuentan los Servicios Sociales se incrementa y expande, en buena parte, hacia servicios institucionalizados (residencias, centros de estancias diurnas...). En cambio, la buena práctica en esta área, que coincide con los deseos de las personas con discapacidad y en situación de dependencia, aconseja concentrar el esfuerzo en la ampliación de las prestaciones para la permanencia en el domicilio (Casado y Fantova, 2007).

Para evitar posibles prácticas erróneas en las asignaciones y propuestas de los recursos y prestaciones, desde nuestro colectivo profesional, debemos tener presente que no toda persona con diversidad funcional (discapacidad) debe ser considerada como una persona en situación de dependencia. Si bien, esto no significa rechazar la relación que pueda existir entre ambas. Esta relación entre diversidad funcional y la situación de dependencia se ha visto influenciada, en gran medida, porque las personas con diversidad funcional han sido discriminadas y minusvaloradas sistemáticamente a lo largo de la historia. Incluso la realidad actual es plenamente discriminatoria y está basada en un conjunto de confusiones conceptuales, muchas de las cuales están relacionadas con la escasa implantación del modelo social y de Vida Independiente en nuestro país.

De este modo, y como se ha visto, la respuesta normativa frente a la situación de dependencia no puede quedar en términos de asistencia, sino que debe ser considerada un derecho de todas las personas a ser protegidas en 
dicha situación, orientando dicho amparo hacia el logro de la autonomía (entendida tanto como el punto de partida cuanto como punto de llegada). Si la falta de autonomía se convierte en un indicio significativo de la existencia de una situación de dependencia, entonces las medidas tendentes a garantizar el mayor grado de autonomía posible serán la respuesta adecuada a ofrecer (De Asís Roig y Palacios, 2007: 56).

En relación a este último aspecto, una de las reivindicaciones más consolidadas por el movimiento de personas con diversidad funcional, y gran desconocido por el colectivo de profesionales del Trabajo Social, es la implementación real de la figura del asistente personal. La aprobación de la Ley 39/2006, de 14 de diciembre, de Promoción de la Autonomía Personal y Atención a las personas en situación de dependencia, establece una prestación económica de asistencia personal, en la que queda reflejada la figura del asistente personal como una prestación más de dicha ley ${ }^{12}$.

No obstante, a pesar de las reivindicaciones del propio colectivo de personas con diversidad funcional en pro de desarrollar una implementación mayor de la figura del asistente personal, lo cierto es que las resoluciones identificadas en el PIA son escandalosamente menores frente a recursos como las residencias ${ }^{13}$.

El enfoque, antes mencionado, de la LAAD hacia un modelo médico-rehabilitador arraigado a prácticas asistencialistas está propiciando el olvido, casi sistemático, de servicios y actuaciones que fomenten la autonomía personal en línea con la filosofía de vida independiente. Sin embargo, en el texto jurídico podemos descubrir los fundamentos específicos de la Filosofía de Vida Independiente, además de en las menciones en el propio título y en la exposición de motivos de la ley, en otras referencias específicas ${ }^{14}$.

No obstante, la implementación real de esta política pública parece estar enfocándose más a la atención de la dependencia que a la promoción de la autonomía. Y, en esta última vertiente los esfuerzos se limitan y conciben como herramientas centradas en características concretas de las personas, $y$ en ningún momento se plantea la posibilidad de que la dependencia tenga además un origen social (De Asís Roig, 2007: 9).

12. Artículo 19 y artículo 2.7.

13. En Andalucía se ha concedido esta prestación sólo en 32 casos frente a 15391 para plazas residenciales (situación a 1 de diciembre de 2009). Extraído el 14 de diciembre de 2009 de http://www.imserso.es/Presentacion/groups/imserso/documents/binario/ prestarecsaad.pdf.

14. Ver artículos 2, 3, 13 y 14. 
En otro lugar, es preciso detenerse sobre la formación adquirida por los trabajadores sociales que estamos desarrollando e implementando las actuaciones derivadas de la LAAD. No podemos negar el valor político de esta ley que ha conllevado una urgencia por incrementar el número de profesionales (aún insuficiente) para hacer frente no sólo a las necesidades de la ciudadanía, sino también a la auto exigencia y expectativas creadas. Un sistema público de estas características debe tener una estructura desarrollada bajo un entorno laboral estable que permita a los profesionales adquirir una formación continua y de calidad en el ámbito en el que se desenvuelven. Sin embargo, en muchos de ellos, se destacan las dificultades y debilidades para analizar e intervenir en los distintos aspectos relacionados con el actual sistema de protección a la dependencia y a las nuevas formas de reorganización del cuidado. Por ello, es muy importante la colaboración entre profesionales, investigadores y teóricos del Trabajo Social, que permita ampliar conocimientos, trasmitir y vincular experiencias, y convertir al Trabajo Social en una materia interesante e imprescindible para los que trabajan en el ámbito del bienestar social (Rossell Poch, 2009).

Dicho todo esto, si miramos la realidad cotidiana de los trabajadores sociales en la actualidad, en los Servicios Sociales comunitarios parece, a juzgar por el número de trámites relativos a las solicitudes para la valoración de la situación de dependencia, y posteriormente por la elaboración de Programas Individuales de Atención, que su única competencia fuera el trabajo relacionado con los trámites del actual sistema de protección social de la dependencia (Villalobos Santos, 2008). Esta situación puede ser percibida como una amenaza para las otras áreas, servicios y programas de los Servicios Sociales comunitarios. Verter todos los esfuerzos humanos, recursos materiales y presupuestarios, a la gestión de las situaciones de dependencia puede hacernos caer en el olvido de otras, y tantas, necesidades sociales que son, y deben ser, atendidas desde los Servicios Sociales comunitarios y por los profesionales del Trabajo Social.

\section{Conclusiones}

La LAAD viene a (re)estructurar la forma institucional de atención a las personas en situación de dependencia. Estas (¿nuevas?) prestaciones y servicios refuerzan, (re)organizan y reproducen, en algunos casos, como hemos visto, las formas tradicionales de provisión del cuidado. En la LAAD, las mujeres, como principales cuidadoras, siguen siendo una figura sin reconocimiento social (en lo referido a la dignificación del trabajo). Esta ley desplaza al colectivo destinatario y lo sitúa como un agente pasivo, receptor de prestaciones 
y recursos (en muchos casos inadecuados, incompletos o mal distribuidos), anulando la posibilidad de participar en su formulación.

El ejercicio profesional del Trabajo Social tiene la responsabilidad de elevar la problemática individual a un nivel social. Se trataría no sólo de implicar al conjunto de la sociedad en las respuestas ofrecidas hacia determinadas necesidades, sino de identificar y elaborar un análisis de la situación de forma holística e integrada, fruto de la sinergia entre el ciudadano (usuario) y el profesional.

Somos conscientes de que en la implementación de la ley los trabajadores sociales sólo formamos una parte de los agentes implicados. No obstante, nuestro ejercicio profesional debe estar en concordancia con los principios de la profesión (justicia social). Así, los retos del Trabajo Social deben pasar por analizar de forma crítica todos los aspectos en los que la profesión entra en contacto con las formas de reorganización del cuidado. Desde esta perspectiva reivindicamos una cohesión profesional que rompa con la excesiva burocratización en las funciones de los trabajadores sociales y que apueste, por el contrario, por el afianzamiento de una disciplina con compromiso profesional y comprometida socialmente. Esta visión puede ofrecer una oportunidad para afrontar los retos y dificultades que la gestión de los cuidados parece estar atravesando.

\section{Bibiliografía}

Agrela Romero, B. y otros, Mujeres inmigrantes en los servicios de atención y cuidado en Jaén. Explorando las condiciones y subjetividades de un trabajo imprescindible e invisibilizado, investigación financiada por la Dirección General de Coordinación de Políticas Migratorias, Jaén, Consejería de Empleo. Junta de Andalucía (2009-2010). 2009a.

Agrela Romero, B., «De los significados de género e inmigración (re)producidos en las políticas sociales y sus consecuencias para la acción e integración social», en Inmigración y Políticas Sociales, L. Cachón, y M. Laparra, (eds.), Barcelona, Bellaterra, 2009b, pp. 239-267.

BARbero, J. M, M. FeU, y A. Vilbrod, La identidad inquieta de los trabajadores sociales, Barcelona, Hacer, 2007.

Alonso Benito, L. E., Trabajo y postmodernidad: El empleo débil, Madrid, Fundamentos, 2000.

- «Los nuevos movimientos sociales en el umbral del año 2000», en Documentación Social, n. ${ }^{\circ}$ 111, Cáritas Española, Madrid, 1998, pp. 155-177.

CASADO, D. y F. FAntova (coords), Perfeccionamiento de los Servicios Sociales en España: Informe en ocasión de la Ley sobre autonomía y dependencia, Madrid, Polibea, 2007. 
Colectivo IOÉ, Mujer, Inmigración y Trabajo, colección Inmigración y Refugio, Madrid, Ministerio de Trabajo y Asuntos Sociales, IMSERSO. 2001.

- «El cuidado de personas mayores dependientes realizado por ciudadanos inmigrantes en la Comunidad de Madrid», en Cuidado a la dependencia e inmigración, Madrid, IMSERSO, 2005, pp. 233-370, [en línea], <http://www. infodisclm.com/documentos/dependencia/depedencia_inmigracion.pdf $>$.

Colectivo Precarias a la deriva, Cuidados globalizados. A la deriva por los circuitos de precariedad femenina, Madrid, Traficantes de sueños, 2004.

COlOM MASFRET, D., «La formación permanente: Retorno con camino eficiente», en Revista de Servicios Sociales y Política Social, Madrid, Consejo General de Colegios de Diplomados en Trabajo Social, 2005. n. ${ }^{\circ} 71$, pp. 23-44.

DE Asís Roig, R., «Reflexiones en torno a la Ley de promoción de autonomía personal y atención a las personas en situación de dependencia», Universitas: Revista de filosofía, derecho y política, Madrid, Instituto de Derechos Humanos «Bartolomé de las Casas», 2007. n. ${ }^{\circ}$ 5, pp. 3-21.

- Derechos Humanos y Situaciones de Dependencia, Madrid, Dykinson, S.L., 2007.

DE La ReD, N., «La formación del Trabajo Social en España», en Revista de Servicios Sociales y Política Social, Madrid, Consejo General de Colegios de Diplomados en Trabajo Social, 1997, n. ${ }^{\circ} 39$, pp. 97-105.

FANTOVA, F., «Algunas notas sobre la identidad profesional en el Trabajo Social», Congreso Estatal del Trabajo Social, Zaragoza, 6 de mayo de 2009, [en línea], <http://www.colegiotstenerife.org/ficheros/File/Congreso-Zaragoza/05_Fernando_Fantova.pdf>.

Foro de Vida Independiente, «Análisis ideológico y de coherencia del anteproyecto de Ley de la promoción de la autonomía personal y atención a las personas en situación de dependencia», 2006, [en línea], <http://www.minusval2000. com/relaciones/vidaIndependiente/lepa/index.html>.

HochSCHILD, R. A., «Las cadenas mundiales de afecto y asistencia y la plusvalía emocional», en En el límite. La vida en el capitalismo global, Anthony Giddiens y Will Hutton (eds), Barcelona, Kriterios Tusquets, 2001, pp. 187-208.

Howe, D., Dando sentido a la práctica. Una introducción a la teoría del trabajo social, Granada, Maristán. 1999.

IMSERSO, Datos sobre prestaciones reconocidas a 1 de Diciembre de 2009, extraído el 14 de diciembre del 2009, [en línea], <http://www.imserso.es/Presentacion/groups/imserso/documents/binario/estsisaad20091201.pdf>.

Las Heras Pinilla, M. P., «Mi experiencia de compromiso con el trabajo social», Servicios Sociales y Política Social, Madrid, Consejo de Colegios de Diplomados en Trabajo Social, 2009. n. ${ }^{\circ}$ 86, pp. 167-172.

Ley 39/2006, de 14 de diciembre, de Promoción de la Autonomía y Atención a las personas en situación de dependencia. 
MARTíneZ BujÁN, R., «Inmigración y envejecimiento desde una perspectiva asistencial», en Género y Empleo, J. Astelarra, (coord.), Madrid, Fundación Carolina, 2009. n. ${ }^{\text {3 } 32, ~ p p . ~ 87-95 . ~}$

Martínez Román, M. ${ }^{a}$ A., M. ${ }^{a}$ T. Mira-Perceval Pastor y H. Redero Bellido, «Sistema público de servicios sociales en España», en Administración Social: Servicios de Bienestar Social, M. ${ }^{a}$ C. Alemán Bracho y J. Garcés Ferrer, (dirs.), Madrid, Siglo XXI Editores, 1996, pp. 203-246.

Moreno Pestaña, J. L., «La demanda de ciencia. Esbozo de una sociología de los discursos epistemológicos en Trabajo Social», en Portularia, n. ${ }^{\circ} 4,2004$, Huelva, pp. 371-386.

Pérez De Orozco, A. y P. BAEZA Gómez, «Sobre -dependencia-y otros cuentos: reflexiones en torno a la ley de promoción de la autonomía personal y atención a las personas en situación de dependencia (Ley 39/2006, de 14 de diciembre, BOE de 15 de diciembre)», en Lan harremanak: Revista de relaciones laborales, n. ${ }^{\circ}$ 15, 2006, Guipuzkoa, pp. 13-40.

PÉrez Orozco, A., «Amenaza de tormenta: la crisis de los cuidados y la reorganización del sistema económico», en Revista de Economía Crítica, n. ${ }^{\circ}$ 5, 2006a, pp. 7-37.

Río, Sira del, «La crisis de los cuidados: precariedad a flor de piel», en Rescoldos, Revista de diálogo Social, n. ${ }^{\circ}$ 9, 2003, pp. 45-57.

Rodríguez Cabrero, G., «La protección social de la dependencia en España. Un modelo sui generis de desarrollo de los derechos sociales», en Política y Sociedad, vol. 44, n. ${ }^{\circ}$, Madrid, 2007, pp. 69-85.

RODRÍGUEZ RODRÍGUEZ, P., «El apoyo informal a las personas mayores en España y la protección social a la dependencia», en Revista española de geriatría y gerontología, vol. 40, extra 3, 2005, pp. 5-15.

Rossell Poch, T., «Una aproximación al Trabajo Social», en Servicios Sociales y Política Social, n. ${ }^{\circ}$ 86, Madrid, Consejo General de Colegios de Diplomados en Trabajo Social, 2009, pp. 163-166.

Vega Solís, C., Cultura del Cuidado en transición, Barcelona, Uoc Editorial, 2009

Villalobos SANTOS, J., «Impacto de la ley de promoción de la autonomía personal y atención a personas en situación de dependencia en los servicios sociales comunitarios», en Documentos de trabajo social: Revista de trabajo y acción social, n. ${ }^{\circ}$ 43-44, 2008, Málaga, pp. 9-28.

Zurdo Alaguero A., «La Dimensión Corporativa del Tercer Sector. Los tipos organizativos del voluntariado», en Revista Internacional de sociología, Vol. LXV, n. ${ }^{\circ} 47,2007$, pp. 117-143. 